\title{
SIMULATIO ET DISSIMULATIO. LE JEU DES ÉMOTIONS DANS LA LITTÉRATURE FRANÇAISE MÉDIÉVALE
}

\author{
Camille CARNAILLE \\ Université de Genève
}

\begin{abstract}
En): This paper deals with the role played by emotions in medieval French literature, especially in this context of simulation or dissimulation. Integrated in the social and courteous norms which rule the society depicted in literature, the emotions need to be controlled and measured, often dissimulated. But this attention paid to the emotions also invites consideration of the larger possibilities of manipulation, and leads to a whole universe of game playing around the emotional expression.
\end{abstract}

Keywords (En): Middle Ages ; emotions ; games ; embodiment ; Tristan

Mots-clés (Fr): Moyen Âge ; émotions ; jeux ; corps ; Tristan

\section{Introduction : De la morale des émotions au Moyen Âge}

L'intérêt des procédés de simulation et de dissimulation n'est plus à démontrer aujourd'hui. Les travaux menés par Jean-Pierre Cavaillé à cet égard ont parfaitement mis en lumière leur importance, notamment au cœur des stratégies de conservation de soi ou de conservation sociale que cet historien décèle au cœur de ce qu'il nomme la civilité au XVII ${ }^{\mathrm{e}}$ siècle (CAVAILLÉ, 2008: 19). Quoique parfaitement convaincue par les analyses et lectures ainsi proposées de ces phénomènes, nous voudrions défendre un ancrage bien plus précoce de cette attention portée à l'extérieur et au paraître, particulièrement des émotions puisque telle est la problématique inscrite au cœur de nos recherches doctorales dédiées aux « jeux des émotions » auxquels fait allusion le titre de cette présentation.

Des nuances sont évidemment à apporter, induites par le prisme que porte le Moyen Âge sur cette notion de la semblance des émotions, nul besoin de réaffirmer ici l'essentielle influence culturelle qui pèse sur l'émotion et plus encore sur sa manifestation au cours de l'histoire, Barbara Rosenwein, Damien Boquet et Piroska Nagy se sont, parmi bien d'autres spécialistes de l'histoire émotionnelle, largement employés à le démontrer ${ }^{1}$.

Ces particularités médiévales sont justement de grand intérêt pour cette thématique de l'apparence, de la simulation et de la dissimulation. Le Moyen Âge est en effet marqué, dans le sillon offert par l'héritage philosophique antique ainsi que par le développement des normes chrétiennes, par une construction morale

\footnotetext{
${ }^{1}$ Nous pouvons à titre tout à fait indicatif noter en effet les importants travaux offerts par ces historiens, véritables moteurs de l'engouement autour de la question des émotions dans le champ de la médiévistique, auteurs notamment des ouvrages de synthèse incontournables : BOQUET Damien et Nagy Piroska (2015), Sensible Moyen Âge. Une histoire des émotions dans l'Occident médiéval, Paris, Seuil, et RosEnwEIN Barbara H. (2015), Generations of Feeling. A history of Emotions 6001700, Chicago, Loyola University Press, parmi bien d'autres recueils, articles et monographies consacrés aux émotions.
} 
très forte des émotions et de leur expression. Les historiens évoqués à l'instant ont bien souligné ces importantes lignes d'influence que constituent les théories platoniciennes, aristotéliciennes ou stoïciennes notamment dans l'élaboration du système médiéval autour des émotions. La pensée chrétienne occupe bien sûr elle aussi une place centrale dans cette réflexion menée sur l'instance affective et oriente sa perception, du vice à la vertu, sans jamais se départir d'une injonction au contrôle et à la méfiance à faire porter sur ces accidents de l'âme ${ }^{2}$.

Dans ce contexte, il convient avant tout de noter le poids exercé par l'idéal d'inspiration aristotélicienne d'harmonie entre intérieur et extérieur, l'apparence étant alors supposée refléter les qualités, ou les défauts, intérieurs. Cet absolu de concordance se voit cependant accompagné d'une réflexion sur le véritable degré de transparence entre ces deux entités (VON MOOS, 1995 : 135), de la conscience d'un fossé entre ces deux pôles de l'homo interior et de l'homo exterior. Ce fossé est tout particulièrement creusé par l'important intérêt témoigné à l'extérieur aussi bien dans une perspective religieuse, animée par un impératif d'introspection et de contrôle hérité de l'apatheia stoïcienne si influente chez les Pères de l'Église, que dans la société laïque et surtout aristocratique qui intègre parfaitement ces préceptes et les adapte à ses idéaux de contenance publique (VON MoOs, 1995 et SCHNELL, 2006). On observe ainsi une oscillation entre authenticité et bienséance (BOUILLOT, 1998 : 112), expression et effacement, spontanéité émotionnelle et silence des figures comme le formulaient Jean-Jacques Courtine et Claudine Haroche dans leur étude de l'expression du visage du $\mathrm{XVI}^{\mathrm{e}}$ au $\mathrm{XIX}^{\mathrm{e}}$ siècle dont nous adapterions volontiers l'objectif ainsi formulé de «faire l'histoire de cette paradoxale injonction à l'authenticité et à la conformité » au Moyen Âge (COURTINE et HaROCHE, $1988: 16$ ).

Cette nécessité de contrôle de soi s'actualise principalement sur le corps qui porte la visibilité et donc le danger social que peut induire la révélation de l'intériorité, comme en témoigne cette réflexion autour de l'homo exterior. Le corps constitue un objet d'analyse d'autant plus intéressant qu'il joue lui aussi d'un dilemme dans la pensée médiévale: il est considéré à la fois comme incontrôlable et comme manipulable (BLANCHFIELD, 2012 et PANCER, 2011). Nira Pancer a bien résumé, dans son éloquent article «Entre lapsus corporis et performance », cette importance de la visibilité des ressentis et surtout du caractère duel du corps comme reflet involontaire de l'intimité et des émotions, mais

\footnotetext{
${ }^{2}$ Il convient de s'arrêter un instant sur la terminologie de cette enquête dont nous souhaitions offrir un aperçu dans cet article. Le terme «émotion » n'apparaît en effet que bien après le Moyen Âge, son utilisation se justifie donc essentiellement dans le cadre du consensus qui semble l'avoir hissé au sein de l'imposant champ de réflexion et de recherche au rang d'étiquette commune et reconnue. Si elle ne connaît pas ce mot "émotion », l'époque médiévale dispose de nombreuses formules pour qualifier ces instances de l'intériorité, celles d'affectus, affectio, ou de passio en latin, ou celles, fréquentes dans la conception médicale, philosophique, mais plus généralement aussi, de mouvement du cœur, d'accident de l'âme, parmi bien d'autres acceptations. Barbara Rosenwein s'est entre autres dédiée à cette problématique lexicale lors du premier colloque organisé par ses collègues Damien Boquet et Piroska Nagy dans le cadre du programme en histoire émotionnelle, EMMA, ou encore lors du colloque organisé autour du sujet des émotions : RosENwEIN Barbara H. (2008), «Emotion words », dans Le Sujet des émotions au Moyen Âge, dir. Damien Boquet et Piroska Nagy, Paris, Beauchesne, p. 93-106.
} 
également comme source de mise en scène, de performance (PANCER, 2011). Lyn Blanchfield a elle aussi exploré ces limites de l'authenticité considérée intrinsèque du corps et ces rituels performatifs auxquels le geste peut alors donner lieu (BLANCHFIELD, 2012), jouant justement sur son aspect profondément révélateur et transformateur (PAGAN, $2011: 255$ ).

Le corps, les gestes et manifestations d'émotions qu'il peut supporter, s'avère d'autant plus important encore dans la pensée et la littérature médiévales que c'est avant tout par son biais que les émotions sont véhiculées. Plusieurs spécialistes de la littérature du Moyen Âge ont déjà pu mettre en lumière cette nette tendance des auteurs à préférer l'expression somatique des émotions à leur description psychique (Bouillot, 1998 et VON MoOs, 1995). Au sein même des théories développées autour des émotions transparaît cette attention pour le corps. Thomas d'Aquin, auteur d'une véritable synthèse des conceptions et systèmes émotionnels au Moyen Âge, souligne le poids de cette essentielle composante psychosomatique de l'instance affective, accorde une grande importance aux données physiologiques des émotions. C'est ainsi que le voile qui se doit de recouvrir les mouvements de l'âme, comme le défendait Carine Bouillot dans un bel article consacré à la pudeur (BOUILLOT, 1998), enveloppe également, voire avant tout le corps comme noyau central du dévoilement de soi. S'élève donc, tout autant qu'autour des émotions elles-mêmes, un éloge de la mediocritas, une devise du retrait en soi pour contrer le caractère révélateur, somatiquement et narrativement, du corps.

Cet idéal de mesure des mouvements de l'âme et de ceux du corps se justifie avant tout par l'intense réflexion menée sur la nature et la valeur des émotions, comme nous l'évoquions. Les passions, telles qu'elles sont le plus souvent nommées dans le système médiéval, sont en effet largement questionnées, dénoncées parfois même comme sources de perturbation de cette contenance visée dans l'idéal chrétien d'élévation vers Dieu, mais aussi dans celui de la convenance dictée par la société aristocratique. Alors fermement condamnées et refoulées, les émotions changent cependant de statut aux abords du XII siècle, sont l'objet d'une attention nouvelle marquée notamment par un retour de la pensée augustinienne qui défendait la portée bénéfique des émotions comme instruments de rapprochement avec Dieu (CASAGRANDE et VECCHIO, 2010: 114). Une nouvelle spiritualité émerge alors, prêtant aux émotions une potentialité vertueuse et un rôle considérable au sein des logiques d'élévation. Cette réhabilitation des émotions ne les libère néanmoins pas de toute contrainte, loin s'en faut. S'il n'est plus question de leur annihilation, c'est leur correcte orientation qui est toujours recommandée, sans que ne disparaisse cette nécessité de maîtrise de soi qui empreint donc l'ensemble de la doctrine médiévale des émotions. Ce principe de contrôle induit bien sûr une prise de conscience des possibilités de manipulation qui y sont liées et mène à une plus large palette de ces jeux des émotions, pas seulement contrôlées, réprimées, dissimulées, mais aussi simulées et mises en scène. Comme l'évoquaient Lyn Blanchfield ou Nira Pancer quant à ses modalités somatiques, l'émotion peut, par sa force expressive clairement reconnue par ces instances de contrôle, s'ériger en véritable outil d'expression, de rituels, de performances qui suscitent et motivent sa manipulation. C'est à ces jeux de 
l'expression émotionnelle que nous voudrions nous consacrer pour envisager l'utilité du secret, de la dissimulation, mais aussi de la simulation, « envisagées comme d'efficaces techniques de gouvernement, mais aussi de réussite sociale ou simplement de protection individuelle, ou encore comme les instruments ordinaires des relations amoureuses »(CAVAILLÉ, 2008 : 22). En reconnaissant ainsi l'efficacité de ces stratégies, Jean-Pierre Cavaillé exacerbe le rôle joué par cette dynamique de l'apparence dans la société qu'il présente d'ailleurs comme « constitutive de la réalité pratique, dès lors que toute stratégie vise à une maitrise des apparences, dans la guerre, en politique, dans la vie de cour ou la séduction amoureuse. » (CAVAillé, 2008: 22) Martine Pagan témoigne déjà de l'importance de ces enjeux dans la société du Moyen Âge en y définissant l'éducation comme l'acquisition d'une contenance (PAGAN, 2011), c'est-à-dire d'une manière d'être, d'un maintien, d'une conduite selon les définitions proposées de cette notion par le dictionnaire de l'ancien français ( $c f$. dictionnaire de Godefroy). La dimension d'utilité sociale et d'efficacité est bien mise en lumière par cette historienne médiéviste qui rejoint ainsi indubitablement les constats posés par Jean-Pierre Cavaillé en soulignant la réalité qu'elle offre et forme ainsi : «Dans la contenance, ce qui est caché s'annule et ce qui est montré fait exister. »(PAGAN, 2011: 256) Ce principe essentiel aux dynamiques de simulation et de dissimulation semble tout particulièrement s'appliquer à l'univers courtois exemplairement mis en scène dans les romans des XII ${ }^{\mathrm{e}}$ et XIII ${ }^{\mathrm{e}}$ siècles. Nombre de spécialistes de la courtoisie ont signalé la place fondamentale du secret, entre maîtrise de soi et dissimulation, au cœur des normes qui animent la société médiévale. Cet impératif concerne au premier rang les amants souvent habités par une véritable obsession de discrétion. De par sa dimension bien souvent adultère, l'amour courtois impose en effet fréquemment le silence, le secret. Mais cette exigence dépasse rapidement la seule raison pratique de préservation liée à l'adultère pour s'immiscer au cœur même des normes courtoises. Associé à une forme d'élitisme amoureux, le secret s'érige ainsi en véritable obligation au sein des arts d'aimer comme en témoigne cet extrait tiré de la traduction en ancien français que propose Drouart la Vache du célèbre De Amore d'André le Chapelain :

Dont, se li dui amant son saige,

Bien celeront en lor coraige

L'amour, touz les jors de lor vie. (DrouART La VACHE, v. 657-659)

Le secret de l'amour est ici explicitement rapproché de la sagesse, ainsi idéalisé et présenté comme indispensable aux conceptions de l'amour courtois. Isabelle Coumert a bien mis en lumière cette composante primordiale du secret comme ressort dramatique majeur des romans bretons, des romans arthuriens notamment, et comme nécessité absolue pour les couples formés par la fin'amor (COUMERT, 2009 : part. 51).

Aux côtés des amants, la figure royale est elle aussi fortement frappée par cette obligation de maîtrise de soi et de performativité sur la scène sociale. Le roi doit en effet se prêter à un important jeu des émotions et apparences, afin de conserver son autorité en démontrant son aptitude au gouvernement de soi et donc des autres, 
mais aussi de l'imposer par une performance de soi et de ses émotions comme la colère, ou la peur, intégrées au cœur de rituels constitutifs de la société courtoise. Plusieurs spécialistes de l'histoire émotionnelle du Moyen Âge ont effectivement pu mettre en exergue cette qualité fondamentale pour un souverain de maîtriser ou de jouer de ses émotions à des fins gouvernementales, citons à seul titre d'exemple l'ouvrage de Laurent Smagghe consacré aux émotions princières (SMAGGHE, 2012). Cette injonction à vocation royale imprègne les manuels de gouvernement qui fleurissent dès le XIII ${ }^{e}$ siècle sous l'étiquette de miroirs aux princes. L'exemple que nous en offre Henri de Gauchy, auteur d'une traduction du fameux traité de Gilles de Rome, est révélateur de cette attention à porter aux émotions et surtout à leur contenance nécessaire en société :

Ceste .x. capitre enseigne, que des movemenz de courage aucuns font a loër et aucuns a blasmer, et enseingne comment les rois et les princes se doivent avenaument contenir es movemenz devant diz. (Li livres du gouvernement des rois, III, X, p. 118, 1. 2-5)

L'association de la contenue à l'avenance, à la convenance transparaît bien dans cet incipit au chapitre que dédie Henri de Gauchy aux mouvements du courage, du cœur, et à leur éducation chez les rois et les princes. Ceci nous ramène parfaitement aux affirmations de Martine Pagan tout en démontrant l'importance de cet idéal de mesure, de retenue qui traverse la pensée médiévale. Il va sans dire que cet appel au secret et au contrôle induit bien d'autres types de jeux émotionnels que ceux de restriction et de retenue, selon un entremêlement des manipulations évoqué plus haut et que nous voudrions à présent développer.

Les romans de Tristan nous offrent un parfait observatoire des dynamiques courtoises et du poids exercé par ces procédés de simulation et de dissimulation. C'est donc à ces œuvres emblématiques de la littérature médiévale que nous voudrions consacrer la brève analyse que nous souhaitions proposer pour illustrer l'importance de ces stratégies au cœur du Moyen Âge.

\section{Les romans de Tristan comme observatoire des jeux des émotions}

La thématique générale de ces romans, la relation adultère de Tristan et Yseut érigée au fil de l'histoire en véritable légende amoureuse et que nous ne ferons donc pas l'affront de représenter, se prête bien sûr parfaitement au motif du secret. Conformément aux topoï de la poétique de la fin'amor élaborée par les troubadours, Tristan et Yseut forment, avec le roi Marc trompé par sa jeune épouse et son neveu, un triangle amoureux qui impose d'emblée la nécessité de cacher, de taire, de manipuler les apparences et particulièrement celle de cet amour coupable. La ruse règne dans ces romans animés par les déguisements, les mensonges et les tromperies, mais une exception pourrait être apportée à son omniprésence en ce qui concerne le jeu émotionnel. Sans s'en départir entièrement bien sûr, les auteurs de la légende tristanienne recourent en effet très peu aux feintises émotionnelles et proposent au contraire un traitement des émotions marqué par sa sincérité. Les mentions de la souffrance des amants par exemple se caractérisent par une grande intensité et ainsi une indéniable véracité de l'émotion exprimée. Se présente toutefois l'une ou l'autre situation lors de laquelle les personnages, et pas 
seulement les amants comme nous pourrions nous y attendre, font preuve de simulation ou de dissimulation de leurs émotions réelles. Mais leur fréquence se révèle plutôt mince en comparaison avec cette multitude de ruses en tous genres mises sur pied par les amants et leurs opposants pour préserver ou dévoiler leur amour. Certaines de ces ruses incluent par ailleurs à l'occasion un recours à la simulation, mais pas spécifiquement d'émotions. Pensons à la scène de faux sommeil de Tristan dans le Roman de Tristan de Béroul (v.760, p.24), ou à celle, célèbre, de Tristan feignant d'être atteint par la lèpre dans ce même roman. Sans aucune ambition d'exhaustivité - qui nous semblait largement dépasser le cadre de cet article -, nous avons sélectionné quelques exemples parmi les plus pertinents et les plus marquants pour tenter de dégager une logique globale de ce recours à la simulation et à la dissimulation dans les romans tristaniens et plus largement peutêtre dans la littérature française médiévale.

\section{- De la ruse de l'adultère}

Notons tout d'abord l'importance de la ruse émotionnelle des amants, bien évidemment poussés par un désir de préservation de leur secret, de leurs sentiments. Curieusement cependant, davantage qu'à une simple dissimulation de leur affection, c'est le plus souvent à de véritables mises en scène d'émotions que se prêtent Tristan et Yseut pour protéger leur relation. Le roman de Béroul, singulièrement animé par cet esprit de ruse que peut impliquer le récit d'un amour adultère, témoigne parfaitement de la feintise émotionnelle requise dans ce contexte. Dès les tout premiers vers conservés du roman, Yseut est ainsi mise en scène simulant la tristesse : «Or fait senblant con s'ele plore. » (BÉROUL, v. 8, p. 1). Nous pouvons tout d'abord noter l'implication physique qui caractérise d'emblée ce jeu émotionnel. C'est en effet par sa manifestation corporelle que s'exprime la tristesse ainsi jouée par Yseut. Cette expression de la tristesse par les larmes renforce évidemment l'ampleur de l'émotion et plus encore de la feintise dont fait preuve Yseut. Nul besoin d'expliciter la forte valeur que confèrent les larmes à ce processus, apparaissant comme le témoin sincère de la douleur, contribuant donc explicitement et efficacement aux enjeux de la simulation. Il s'agit là d'un paradigme commun au sein des jeux des émotions qui recourent volontiers au corps, souvent considéré intrinsèquement authentique et honnête dans la pensée médiévale, pour mieux convaincre des émotions mises en scène. Tous les cas de feintise ne poussent pas aussi loin la démarche, l'implication physique de la simulation, mais il est intéressant de constater que de nombreuses occurrences incluent ainsi le corps, le visage du moins. C'est à nouveau le cas dans le même roman de Béroul lorsque Tristan, déguisé en lépreux, affecte la douleur en portant la reine sur son dos :

L'un pié sorlieve et l'autre clot :

Sovent fait senblant de choier,

Grant chiere fait de soi doloir. (BÉROUL, v. 3940-3942, p. 122)

Tout le corps est ici engagé dans la ruse de Tristan, puisqu'il mime aussi bien la chute qu'il ne tord son visage d'une douleur tout apparente. Ce passage nous 
renseigne en outre sur la fonction importante que peuvent occuper ces simulations d'émotions dans la trame du roman. Cette scène quasi théâtrale s'insère en effet dans le cadre de la ruse plus générale, et bien plus essentielle à l'histoire des amants, d'Yseut, qui recourt à ce stratagème de se faire porter par Tristan, rendu méconnaissable par son apparence de lépreux, pour pouvoir honnêtement jurer n'avoir eu entre ses cuisses d'autres hommes que le roi et cet homme qui l'a portée juste auparavant. La visée utilitaire de cette feintise émotionnelle apparaît très clairement ici, puisqu'elle permet à la reine, à l'heure de ce quasi-procès qui lui est imposé, de se voir lavée de tout soupçon quant à son présumé adultère, mais aussi ainsi de le poursuivre. Nous rejoignons en ceci les analyses proposées par Insaf Machta de l'univers tristanien : «La ruse, qui est souvent au service de cette quête [de la proximité physique], se présente comme le moyen, souvent incontournable, de l'actualisation du désir » (MACHTA, 2010 : 149). Uniquement animés par ce désir, Tristan et Yseut ne semblent recourir à ces manipulations émotionnelles qu'à la seule fin d'en assurer la réalisation dans cette proximité physique tant recherchée, évidente dans un contexte amoureux et plus encore quand elle se voit problématisée dans ce cadre d'amour adultère.

\section{- Du secret de la fin'amor et du ressort de protection des jeux émotionnels}

Quoique prépondérant, cet objectif n'est cependant pas le seul poursuivi au sein des romans tristaniens, pas même par les amants qui jouent aussi de leurs émotions au nom du secret qui se doit d'entourer leur relation. Outre l'intensité, le caractère irrépressible qui entoure le plus souvent leurs sentiments, l'amour de Tristan et Yseut se conçoit en effet essentiellement dans le cadre de sa dimension adultère. Mais bien autrement qu'une ruse explicite, ceci semble avant tout motiver et justifier un besoin de taire, de cacher, de dissimuler les apparences, et surtout celle de cet amour coupable. C'est particulièrement le cas de Tristan une fois marié à Yseut aux Blanches Mains dans le roman de Thomas. Alors exilé loin de la cour de son oncle, le roi Marc, et de la belle Yseut la Blonde qui continue de faire battre son cœur en dépit de son union factice, Tristan éprouve la plus grande des difficultés à dissimuler la souffrance de cette séparation et plus généralement ses sentiments à sa nouvelle épouse :

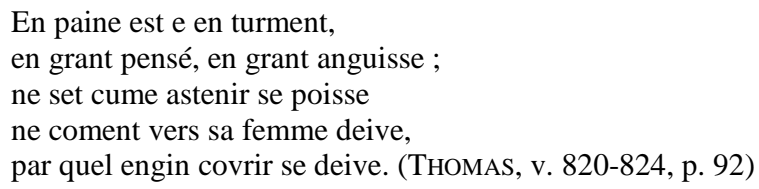

On perçoit bien ici l'angoisse de Tristan, étreint par le souvenir d'Yseut, et le dilemme qui se pose alors à lui, entre la promesse faite à l'amante, de ne jamais se voir préférée, et celle, bien évidente, à l'épouse, celle de l'amour et celle du mariage. L'insistance sur la grande souffrance ressentie, répétée à quatre reprises sur deux vers seulement, exacerbe l'embarras perceptible de Tristan pour dissimuler ses tourments. Cette dissimulation est néanmoins présentée comme 
impérative à mettre en œuvre par la répétition du verbe devoir, ainsi que par la mention à l'abstention, à la contenue, qui soulignent l'importance de cette obligation fondamentale du secret, de la retenue qui frappe les amants. Le terme engin, qui désigne en ancien français l'habileté, l'adresse, plus spécifiquement la ruse, la fraude, la tromperie, l'artifice ( $c f$. dictionnaire de Godefroy), met en lumière la dimension effectivement manipulatrice de cette dissimulation. Le texte revient un peu plus loin sur cette nécessité de dissimulation, aussi malaisée soitelle, pour dépeindre les efforts, toujours confus, de Tristan pour y parvenir :

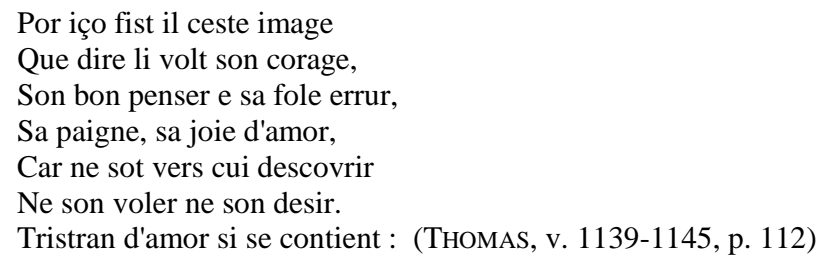

Le narrateur nous relate ainsi le recours de Tristan à une statue auprès de laquelle il peut laisser libre cours à sa peine et à ses doutes, à défaut de confident à qui il pourrait honnêtement ouvrir son cœur. On note ici l'entremêlement d'émotions éprouvées et dès lors dissimulées à tout son entourage par Tristan: amour, regret, peine, joie, désir. L'acte créateur qui est à l'origine de la statue et donc de la confidence, bien explicité en début de citation, appuie le désir de dissimulation et sa difficulté persistante, en démontrant les efforts fournis par Tristan, les réalisations qui lui ont été nécessaires pour parvenir à garder cachées ses émotions, à les contenir. À nouveau, il n'est guère compliqué de deviner le but poursuivi par Tristan au gré de cette dissimulation ni surtout la raison qu'a le narrateur de l'introduire, le dénouement du roman se basant essentiellement sur le silence de ses émotions de la part de Tristan.

C'est néanmoins la propre ruse d'Yseut aux Blanches Mains, toujours dans le roman de Thomas, qui se révèle la plus représentative de cet enjeu sous-tendu derrière la simulation et la dissimulation d'émotions. C'est surtout lorsqu'elle apprend finalement la cause de la distance de son époux à son égard, l'amour qu'il porte à Yseut la Blonde, qu'Yseut se prête le mieux à ce jeu de manipulation qui conduira in fine Tristan et son amante à leur perte :

\footnotetext{
Ysolt est en la chambre entree, vers Tristan ad s'ire celee, sert le e mult li fait bel semblant cum amie deit vers amant. Mult ducement a li parole e sovent le baise e acole, e mustre lui mult grant amur e pense mal en cele irrur par quel manere vengee ert, e sovent demande e enquert kant Kaherdin deit revenir od le mire qu'il deit guarir. De bon curage pas nel plaint, la felunie el cuer li maint
} 
qu'ele pense faire, s'ele puet,

car ire a iço la comuet. (THOMAS, v. 2773-2788, p. 212)

On observe ici un double processus, une manipulation totale des émotions, passant aussi bien par une dissimulation de son ire, de sa colère, que par une simulation, longuement démontrée, de bienveillance, de mult grant amur même. Cette intensité de l'amour ainsi manifesté exacerbe bien sûr la teneur de la feintise, mais aussi le fossé qui s'installe entre l'apparence et les sentiments réels d'Yseut. Le narrateur prend d'ailleurs bien soin de préciser ensuite le désir de vengeance qui étreint Yseut et combien son cœur, bien autrement que d'amour, est empli de felunie. La répétition de la colère qui la comuet, l'émeut, la soulève ( $c f$. dictionnaire de Godefroy), vient finalement insister sur les émotions véritablement ressenties par Yseut, cette ire qui conduira alors tous ses actes. Cette colère et son camouflage se révèleront en effet capitaux pour la suite du récit: Tristan ignorant tout des nouvelles dont fut informée son épouse et de l'hostilité que celles-ci suscitèrent en elle, il ne se méfiera en rien de son comportement et de ses mensonges, particulièrement celui, célèbre, proféré quant à la couleur de la voile du bateau amenant Yseut la Blonde. Plus encore, c'est sa simulation de bienveillance qui induira Tristan en erreur en endormant sa vigilance, le narrateur insistant ainsi sur sa dimension véritablement rusée, notamment par l'opposition créée entre l'image d'amour qu'Yseut renvoie et la félonie qui lui emplit en réalité le cour.

Ce mélange tout à fait particulier de simulation et de dissimulation s'avère assez fréquent surtout chez le personnage du roi Marc dans Le Roman de Tristan en Prose, dans une tout autre optique alors. À de nombreuses reprises, Marc est dépeint comme éprouvant une émotion de haine, de colère, notamment face à Tristan, qu'il réprime et surtout que vient recouvrir une apparence bienveillante, de joie, voire d'amour: «Mout est li rois Mars dolenz de ceste novele, et neporquant si fait il semblant de joie por ce que nus de leanz ne s'en aperceüst. » (Le Roman de Tristan en Prose, t.2, 483, 10-12, p. 91). La dimension sociale motivant la simulation est parfaitement intégrée ici, témoigne de cette performativité émotionnelle requise sur la scène publique. La société courtoise du Moyen Âge, particulièrement celle transposée en littérature, étant profondément empreinte des règles de bienséance et d'honneur, s'observe un véritable processus de mise en scène de la sociabilité courtoise. Dans ce contexte, les émotions s'avèrent tout logiquement elles aussi influencées par la conscience élevée du jeu public, du poids de la sphère sociale. Ceci expliquerait donc le caractère policé démontré, dans cet extrait et dans bien d'autres, par le roi Marc dans la manifestation de ses émotions, dirigées par un sens accru de l'affabilité et surtout des convenances en public. Il est assez clair ici que si Marc ne consent pas à l'expression de ses émotions, les dissimule, en simule d'autres pour mieux les recouvrir, c'est avant tout pour ne pas quitter sa posture royale et ce qu'elle implique. Céder à la colère, faire montre de tristesse en pleine cour ne saurait en effet correspondre aux normes courtoises auxquelles le roi, plus que quiconque, doit se conformer. Cette démarche pourrait en quelque sorte se voir opposée à celle des amants, que l'amour détourne, selon un schéma assez courant dans la 
littérature médiévale, de la raison sociale. C'est particulièrement dans cette perspective courtoise, au-delà du contexte d'amour caché, que s'observent dans les romans de Tristan davantage de dissimulations que de simulations explicites. Cette constatation rejoint donc notre première appréhension du corpus tristanien, certes centré sur la ruse dans sa thématique générale, mais pas spécifiquement quant à son traitement des émotions, dans lequel pointe une relative impression d'honnêteté, de conformité tout au moins. La dimension de ruse motivée par le caractère adultère de l'amour que se portent Tristan et Yseut transparaît néanmoins dans leur propre comportement émotionnel, eux qui simulent presque plus qu'ils ne dissimulent, dans une volonté de tromperie bien plus assumée que celle des autres personnages de ces récits. On ne note en effet aucune mention de simulation d'émotions de la part des nombreux opposants des amants, pourtant eux aussi prodigues en ruses et fourberies pour tenter de les piéger. Et s'il arrive à Marc de simuler, nous avons vu que ses artifices se révélaient habités des plus nobles intentions : s'en tenir à son statut de roi et assurer la valeur de sa cour. La faible proportion de simulations et leur cantonnement aux amants - distraits, comme on l'a vu, des règles courtoises - tendraient à traduire le caractère assez réduit de la dimension de ruse à proprement parler, la feintise étant davantage dictée par la pression sociale que par une réelle volonté de tromperie. Nous aboutissons donc aux mêmes conclusions que celles établies par Jean-Pierre Cavaillé quant à cette importante dimension de civilité qui entoure et motive la simulation et la dissimulation.

\section{Conclusion : Esquisse de la simulation et de la dissimulation dans la littérature médiévale}

Sans prétendre avoir esquissé un véritable modèle des jeux émotionnels dans la littérature médiévale, nous pouvons déjà dégager de ces quelques exemples d'intéressants constats autour du traitement des émotions et particulièrement de leurs manipulations. Une très rapide comparaison des trois œuvres présentées pourrait nous offrir un condensé des dynamiques qui les entourent. Nous pouvons en effet noter les particularités de la narration de Béroul qui joue le mieux de cet univers de ruse comme on a pu le percevoir au travers des deux extraits analysés, animés par une dimension trompeuse indéniable. Au contraire, le roman de Thomas semble davantage empreint de cette dynamique courtoise de maintien de soi, de conformité à la bienséance, tout comme Le Roman en Prose particulièrement sensible aux impératifs de la posture royale de Marc déchiré entre ses émotions et ses obligations. On obtient ainsi un certain balisage des logiques à l'œuvre au sein des procédés de simulation et de dissimulation, marqués tout d'abord par la diversité des objectifs poursuivis : la dissimulation tendrait avant tout à la protection de soi et au respect des bienséances, tandis que la simulation semble au service d'une ruse plus explicite quand elle n'assure pas l'efficacité de ladite protection. Notons aussi les caractéristiques liées à l'implication du corps renforçant particulièrement la simulation d'émotions pour mieux la mettre en scène, au sens social engagé - réponse aux convenances ou contournement de celles-ci -, mais aussi aux multiples critères auctoriaux et narratifs en jeu, 
démontrant parfaitement l'intérêt et l'importance de ces questions de simulation et de dissimulation dans la littérature.

\section{BIBLIOGRAPHIE}

\section{Corpus d'analyse}

BÉrOUl, Le Roman de Tristan, éd. Ernest Muret (1903), Paris, Firmin Didot.

Drouart LA VACHE, Li Livres d'Amours, éd. Robert Bossuat (1926), Paris, Champion.

Thomas, Le Roman de Tristan. Suivi de La Folie Tristan de Berne et La Folie Tristan d'Oxford, éd. Félix Lecoy, trad. Emmanuèle Baumgartner et Ian Short (2003), Paris, Champion.

Le Roman de Tristan en Prose, éd. Renée Curtis (1976), t.2, Leiden, Brill.

Li livres du gouvernement des rois : a XII ${ }^{\text {th }}$ century French version of Egidio Colonna's treatise de Regimine principum, éd. Samuel Paul Molenaer (1899), Londres, MacMillan \& Co.

\section{Littérature secondaire}

Bestul Thomas H. (1983), True and false cheere in Chaucer's Clerk's Tale, Journal of English and German Philology 82/4, p. 500-514.

BLANCHFIELD Lyn (2012), Prolegomenon. Considerations of Weeping and Sincerity in the Middle Ages, in : GerTsman Elina (éd.), Crying in the Middle Ages. Tears of History, New York/Londres, Routledge, p. xxi-xxx.

BOLENS Guillemette (2008), Le Style des gestes. Corporéité et kinésie dans le récit littéraire, Lausanne, BHMS.

BoQUET Damien ; NAGY Piroska (2015), Sensible Moyen Âge. Une histoire des émotions dans l'Occident médiéval, Paris, Seuil.

BouILlot Carine (1998), Gestes et pudeur dans les romans courtois du XIII ${ }^{\mathrm{e}}$ siècle, in : Le Geste et les gestes au Moyen âge, Aix-en-Provence, CUERMA, p. 109-124.

BOUREAU Alain (2007), La passion et la faute. Nouvelles approches des émotions médiévales, Critique 716-717, p. 36-46.

CASAGRANDE Carla ; VeCCHIO Silvana (2010), Les théories des passions dans la culture médiévale, in : BoQUET Damien et NAGY Piroska (éd.), Le sujet des émotions au Moyen Âge, Paris, Beauchesne, p. 107-122.

CAVAILlÉ Jean-Pierre (2008), Dis/simulations : Jules-César Vanini, François La Mothe Le Vayer, Gabriel Naudé, Louis Machon et Torquato Accetto. Religion, morale et politique au XVII siècle, Paris, Champion.

CAVAILlÉ Jean-Pierre (2006), Tous les secrets du monde, Sigilia 17.

CERQUiglini Jacqueline (1988), Polysémie, ambiguïté et équivocité dans la théorie et la pratique poétiques du Moyen Âge français, in : ROSIER Irène (éd.) L'Ambiguïté. Cinq études historiques, Lille, Presses Universitaires de Lille, p. 167-184.

COILLY Nathalie ; TESNIÈRE Marie-Hélène (dir.) (2012), L'art d'aimer au Moyen $\hat{A} g e$. Le Roman de la rose, Paris, Bibliothèque Nationale de France. 
COUMERT Isabelle (2009), « Si ceste amur esteit seüe... ». L'obligation du secret dans la fin'amor (XII'-XIII' siècles), Questes 16, p. 51-63.

Courtine Jean-Jacques ; HAROCHE Claudine (1988), Histoire du visage. Exprimer et taire ses émotions $\left(X V I^{\mathrm{e}}-X I X^{\mathrm{e}}\right.$ siècle), Paris, Rivage.

DEMBOwsKi Peter F. (1988), Le Faux Semblant et la problématique des masques et des déguisements, in : OLLIER Marie-Louise (éd.), Masques et déguisements dans la littérature médiévale, Paris/Montréal, Vrin/Presses Universitaires de Montréal, p. 43-53.

EMING Jutta (2006), Affektüberwältigung als Körperstil im höfischen Roman, in : PHILIPOWSKI Katharina et PRIOR Anne (éd.), Anima und sêle. Darstellungen und Systematisierungen von Seele im Mittelalter, Berlin, Eric Schmidt Verlag, p. 249-262.

FRIEDMAN Lionel J. (1989), La Mesnie Faux Semblant : Homo interior =/= Homo exterior, French Forum 14, p. 435-445.

GALLY Michèle (1997), Chanson de geste et roman : stylisation de l'émotion et écriture singulière, in : Coudreuse Anne et DELIGNON Bruno (éd.), Passions, Emotions, Pathos, Rennes, Presses Universitaires de Rennes, p. 7-22.

GALlY Michèle (2005), L'intelligence de l'amour d'Ovide à Dante. Arts d'aimer et poésie au Moyen Âge, Paris, CNRS.

LE GoFf Jacques ; TRUONG Nicolas (2003), Une histoire du corps au Moyen Âge, Paris, Liana Levi.

MACHTA Insaf (2010), Poétique de la ruse dans les récits tristaniens français du $X I I^{\mathrm{e}}$ siècle, Paris, Champion.

NASH Penelope (2015), Reality and Ritual in the Medieval King's Emotions of Ira and Clementia, in: CHAMPION Michael et LYNCH Andrew (éd.), Understanding Emotions in Early Europe, Turnhout, Brepols, p. 251-272.

PAGAN Martine ; THOMASSET Claude (éd.) (2011), Cacher, se cacher au Moyen Âge, Paris, Editions Paris-Sorbonne.

PANCER Nira (2011), Entre lapsus corporis et performance : fonctions des gestes somatiques dans l'expression des émotions dans la littérature altimédiévale, Médiévales 61, p. 39-54.

RASMUSSEN Ann Marie (2003), Emotions, Gender, and Lordship in Medieval Literature : Clovis's Grief, Tristan's Anger, and Kriemhild's Restless Corpse, in : JAEGER Stephen C. et KASTEN Ingrid (éd.), Codierung von Emotionen im Mittelalter, Berlin, De Gruyter, p. 174-189.

REVEST Clémence (2009), Conclusion: Pour vivre heureux, vivons cachés ?, Questes 16, p. 84-86.

SCHMITT Jean-Claude (1987), La morale des gestes, Communications. Parure, pudeur, étiquette 46, p. 31-47.

SCHNELL Rüdiger (2006), Wer sieht das Unsichtbare ? Homo exterior und homo interior in monastichen und laikalen Erziehungsschriften, in : PHILIPOWSKI Katharina et PRIOR Anne (éd.), Anima und sêle. Darstellungen und Systematisierungen von Seele im Mittelalter, Berlin, Eric Schmidt Verlag, p. 83-112.

SMAGGHE Laurent (2012), Les émotions du Prince, Paris, Garnier. 
SOLTERER Helen (1984), Le bel semblant. Faus Semblant, Semblants romanesques, Médiévales 3/6, p. 26-36.

TANASE Gabriela (2003), De Tristan à Villon, les ruses du masque et du discours, mémoire de master présenté sous la direction de M. Jeay, Hamilton, McAster Université.

Von Moos Peter (1995), Occulta cordis. Contrôle de soi et confession au Moyen Âge I. formes du silence, Médiévales 29, p. 131-140.

Von Moos Peter (1996), Occulta cordis. Contrôle de soi et confession au Moyen Âge (suite), Médiévales 30, p. 117-137. 